\title{
Small Polymeric Toys Placed in Child-Dedicated Chocolate Food Products-Do They Contain Harmful Chemicals? Examination of Quality by Example of Selected VOCs and SVOCs
}

\author{
Monika Śmiełowska ${ }^{1}$. Mariusz Marć ${ }^{1} \cdot$ Bożena Zabiegała ${ }^{1}$
}

Received: 12 April 2021 / Revised: 19 August 2021 / Accepted: 30 August 2021 / Published online: 12 September 2021

(C) The Author(s) 2021

\begin{abstract}
This study was focused on child-dedicated chocolate food products that contain a plastic package with a small toy inside (also known as chocolate eggs). Three types of these products that are commercially available on the Polish market, with different prices, chocolate composition, and main types of polymers used in the toy manufacturing process, were investigated. The polymers were identified using Fourier-transform infrared (FT-IR) analysis and emissions of selected toxic volatile organic compounds of toy and packaging samples were identified using stationary emission micro-chamber. Total volatile organic compounds emissions were also estimated. Moreover, the content of polybrominated diphenyl ethers (PBDEs) as bioaccumulative semi-volatile organic compounds was checked in all parts of the cheapest product. The highest PBDE concentrations, which ranged from $5.32 \pm 0.79$ to $1768 \pm 289 \mathrm{ng} / \mathrm{g}$, were recorded in chocolate samples. This demonstrates the need for consumer knowledge on the safety of such products available on the Polish market to be broadened. This is one of the many examples of studies showing that products sold on the European market do not always comply with the Conformité Européenne (CE) declaration of conformity. Furthermore, the presence of phased-out PBDEs in toys may provide evidence of wrong recycling practices. Forward-looking considerations showed that, based on assessments of the hazard quotient (HQ) and cancer risk of BDE-209 and assuming each child has contact with one toy and eats one of the studied chocolate products per day, there is no high risk associated with the exposure of children to PBDEs.
\end{abstract}

Keywords Monoaromatic hydrocarbons · Total volatile organic compounds (TVOC) · Polybrominated diphenyl ethers (PBDEs) $\cdot$ Chocolate eggs safety $\cdot$ Exposure assessment

\section{Introduction}

Plastic materials are considered some of the main sources of the emission of volatile and semi-volatile organic compounds (VOCs and SVOCs, respectively) into the environment (Noguchi and Yamasaki 2020). Emission of these chemicals is a very important phenomenon, especially in the case of synthetic polymeric materials and products that have direct contact with food products (food contact materials, FCMs), due to the possibility of chemicals directly migrating from these plastic materials into food products

Bożena Zabiegała

bozena.zabiegala@pg.edu.pl

1 Department of Analytical Chemistry, Faculty of Chemistry, Gdańsk University of Technology (GUT), Narutowicza 11/12 St., 80-233 Gdańsk, Poland
(Szczepańska et al. 2018; Bauer et al. 2019; Martinez-Bueno et al. 2019). Accordingly, there are legislative requirements for polymers intended to be in contact with food. In Member States of the European Union (EU), this is primarily controlled by Commission Regulation (EU) No. 10/2011 on plastic materials and articles. This regulation established rules for the composition of plastic FCMs, and provides a Union List of substances permitted for use in the manufacturing of plastic FCMs. It also sets out restrictions on the use of these substances, and lays down rules for the compliance of plastic materials and articles (European Union 2011).

The presence of VOCs and SVOCs in polymeric materials mainly occurs by the following routes: (i) residues from the polymer synthesis process (residues of polymerisation, or traces of the initiators, catalysts, or solvents that were used therein); and (ii) substances introduced in the manufacturing process of plastic materials (antioxidants, plasticisers, UV stabilisers, as well as flame retardants (Hankett et al. 2013; 
Pei et al. 2017; Azwa et al. 2013; Mitchell et al. 2014; Kozai et al. 2018; Yanagisawa et al. 2018). In addition, various compounds can be formed and released due to structural degradation of the polymeric material, for example, after exposure to high temperatures and/or UV light (Royaux et al. 2017; Formela et al. 2017; Yu et al. 2016; Bhaskar et al. 2007). VOCs are released from the surface of indoor materials made of polymeric materials as a consequence of application various types of solvents, reagents and polymer monomers during their manufacturing process. Following the literature data, the emission profile, as well as the type and the amount of liberated VOCs, might be associated with the composition and morphological characteristics of defined plastic material. For this reason, the emission determination for VOCs representatives such as monoaromatic hydrocarbons might be considered as a preliminary assessments of the type and quality of polymeric materials associated with the potential solvents and unreacted monomer residues in them (Mitchell et al. 2014; Lattuati-Derieux et al. 2013; Wilke et al. 2004). In terms of polymer safety, important representatives of monoaromatic hydrocarbons are benzene, toluene, ethylbenzene, xylenes (BTEX) and styrene. These compounds are known or suspected to cause cancer or other serious health effects (Mitchell et al. 2014). Another valuable parameter is total volatile organic compounds (TVOC) emission. On the other hand, one class of SVOCs that may be found in polymeric materials are polybrominated diphenyl ethers (PBDEs), which have been used in the past as flame-retardant additives (Rahman et al. 2001). The basic international agreement that includes PBDEs in the group of persistent organic pollutants (POPs) is the Stockholm Convention. Commercial mixtures of PBDEs are listed under Annex A of this Convention, which specifies substances whose production and use should cease completely (Tang 2013). The Stockholm Convention has resulted in the EU Directive on the Restriction of Hazardous Substances (Directive 2015/863/EC 2015; Xu et al. 2013). This document defines the maximum concentrations of PBDEs (established at $1000 \mathrm{ppm}$ ) permitted to be included in the homogeneous material of articles intended for daily use. A related document is the Toy Safety Directive (2009/48/EC), which requires manufacturers to carry out a risk analysis before placing a toy on the market, and to assess the possible exposure of children to such risks (Directive 2009/48/ CE 2009). According to this Directive, toys placed on the European market must have a declaration of conformity, a legally binding promise stating that the product complies with European law. Before the manufacturer issues a declaration of conformity, products should undergo a conformity assessment procedure and obtain the appropriate certificates. If the product receives a declaration of conformity, the manufacturer marks it with the Conformité Européenne (CE) seal, allowing it to be sold within the European Economic Area (Directive 2009/48/CE 2009).

The key issue here appears to be the recycling of polymers, which manufacturers are currently pursuing. Recycled polymers must undergo appropriate removal of hazardous compounds to be reused, particularly in "sensitive use" areas (toys, food packaging, food containers, kitchen equipment, water tanks, and water pipes) (UNEP 2017). Despite the abovementioned regulatory activities, the issue of the occurrence of PBDEs in the environment has not been resolved because they are persistent and can bioaccumulate (Currier et al. 2020; Wu et al. 2020; Kelly et al. 2008). In addition, there are reports that imported consumer goods do not always meet the European requirements regarding PBDEs (Ionas et al. 2014). This is a very important issue, especially in the case of plastic materials or products that are intended for use by or have direct contact with children, as this could lead to child exposure to PBDEs. Children have been shown to be the social group the most strongly affected by exposure to PBDEs, and adverse health effects impact prenatal and small children particularly strongly (Vuong et al. 2018a, b, c). PBDEs have been reported to act as endocrine disruptors (Ji et al. 2019; Vuong et al. 2018a, b, c) and can cause neurological defects (Drobna et al. 2019), permanent learning and memory impairment (Cowell et al. 2018), behavioural changes (Vuong et al. 2017, 2018a, b, c), hearing defects (Fabelova et al. 2019), delayed onset of puberty (Harley et al. 2017), decreased sperm count (Zhang et al. 2020), foetal malformations (Koren et al. 2019), and, possibly, cancer (Hoffman et al. 2017). The main routes of exposure to PBDEs are the oral route, with the most significant sources listed as: dust, meat and dairy products (Śmiełowska and Zabiegała 2020; Pietron et al. 2021; Zacs et al. 2021; Konstantas et al. 2018).

In this study, we evaluated the content of BTEX, styrene, TVOCs and 8 representative PBDEs in the different parts of 3 types of commercially available child-dedicated chocolate food products inside which toys are placed (i.e., chocolate eggs containing a polymeric toy inside a polymeric package). The data about the type and amount of detected compounds were combined with the results of Fourier-transform infrared spectroscopy (FT-IR) analysis. To the best of our knowledge, this is the first study to combine the testing of this type of product for VOCs and SVOCs, in parallel. In particular, this is the first study of the presence of PBDEs in such products. Therefore, to elaborate on the issue of the presence of PBDEs, we performed forward-looking considerations involving theoretical estimation of child exposure in contact with the tested product. All available routes from the parts of the products being tested, i.e., the chocolate egg (by ingestion) and polymeric toy or package (by inhalation, dermal contact, and hand-to-mouth ingestion) were included. 


\section{Materials and Methods}

\section{Analyzed Samples}

Commercially available child-dedicated chocolate eggs with a small toy inside manufactured by three different companies were selected for use in this study $(n=8$ for each product type). For each of the three product types, products from the same production series were selected. Figure 1 shows the parts of the commercially available child-dedicated chocolate food products studied herein.

Many types of chocolate children's food products with a small toy inside are available on the Polish market. They mainly differ in the following ways:

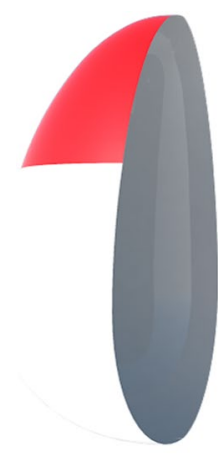

A

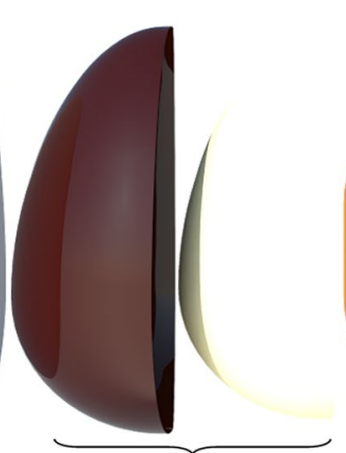

B
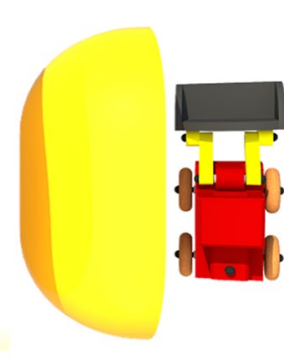

C
- the use of single polymers or mixtures of polymers with different physicochemical properties for the production of toys, as some toys have significantly greater hardness, elasticity, and deformability than others;

- type of toy: toys can either consist of several elements to be assembled, or one-piece toys (small figurines) can be included;

- different toy packaging: products are available on the market with transparent packaging or completely nontransparent packaging, and sometimes there are differences in the way the boxes are opened and in their manufacturing quality;

- different cocoa mass content in the composition of the chocolate, which in turn affects the fat mass of the chocolate and the perception of the taste of the chocolate, with products with a higher cocoa mass content characterised by higher prices and perceived as having better taste by consumers;

- where the chocolate and toy are produced, both are not always produced in the same country; and

- the price of the product, reflecting the quality of both the toy and chocolate.

Table 1 presents general information on the tested products, which are commercially available on the Polish market. In this study, 8 pieces of each product (marked as _1, 22 , and _ 3 in Table 1) from the same production batch were used. Calculation of the mean PBDE concentration for each of the three product types tested was possible due to the fact that products from the same production batch were used in the study.

Samples were purchased at local stores in Gdansk, Poland. Before analysis, they were stored at room

Fig. 1 Parts of a chocolate children's food product with a polymeric toy placed inside: A aluminum foil cover; $\mathbf{B}$ layers of chocolate; $\mathbf{C}$ polymeric package for toy; $\mathbf{D}$ polymeric toy

Table 1 Basic information about the commercially available child-dedicated chocolate food products containing small polymeric toys examined in this study

\begin{tabular}{|c|c|c|c|}
\hline Parameter & Product_1 $(n=8)$ & Product_2 $(n=8)$ & Product_3 $(n=8)$ \\
\hline Mean chocolate mass $\pm \mathrm{SD}(\mathrm{g})$ & $20.95 \pm 0.65$ & $21.2 \pm 1.1$ & $26.2 \pm 1.6$ \\
\hline $\begin{array}{l}\text { Mean polymeric package } \\
\text { mass } \pm \mathrm{SD}(\mathrm{g})\end{array}$ & $3.940 \pm 0.088$ & $3.899 \pm 0.096$ & $3.814 \pm 0.085$ \\
\hline Mean toy mass $\pm \mathrm{SD}(\mathrm{g})$ & $5.9 \pm 1.3$ & $3.17 \pm 0.77$ & $0.720 \pm 0.062$ \\
\hline $\begin{array}{l}\text { Assumed total surface area of the } \\
\text { toy }\left(\mathrm{cm}^{2}\right)\end{array}$ & 10 & 10 & 10 \\
\hline Fat content in the chocolate & $34.1 \%$ & $31.5 \%$ & $31.7 \%$ \\
\hline Appearance of the toy packaging & Non-transparent, yellow & Transparent, white & Non-transparent, yellow \\
\hline General characteristics of toys & Hard material, toys to be assembled & Hard material, toys are figurines & $\begin{array}{l}\text { Flexible material, toys to be } \\
\text { assembled }\end{array}$ \\
\hline Product price & The highest-priced original product & Medium-priced original product & The lowest-priced original product \\
\hline CE label & Yes & Yes & Yes \\
\hline Intended age group & $>3$ years & $>3$ years & $>3$ years \\
\hline Origin of the chocolate & EU & EU & Turkey \\
\hline Origin of the toy & EU & China & China \\
\hline
\end{tabular}


temperature, similar to what happens with products on store shelves. Before extraction, the chocolate samples were manually broken up into small, irregular pieces.

Polymer samples (of both toys and packages) were cut into small pieces with scissors. The scissors were washed with acetone between different samples.

\section{FT-IR Analysis}

To identify the main type of polymers used in the manufacturing process of the small plastic toys and plastic packages placed in the studied chocolate products, Fourier-transform infrared spectroscopy (FT-IR) was used. The FT-IR analysis of samples was implemented with a Nicolet Spectrometer IR200 obtained from Thermo Scientific (Waltham, MA, USA). This device had an attenuated total reflection (ATR) attachment with a diamond crystal. Measurements were performed with $1 \mathrm{~cm}^{-1}$ resolution over the wavenumber range from 4000 to $400 \mathrm{~cm}^{-1}$ under 64 scans (Przybysz et al. 2018). The results obtained for the studied samples of small toys and plastic packages were compared to the reference spectrum enclosed in the software database attached to the ATR-FT-IR device (Hummel Polymer Sample Library) The identification of the main type of polymer from which the investigated small toys samples were made was based on the interpretation of the spectrum obtained for the sample in comparison with the spectrum available in the software database (fit values above $92 \%$ were adopted as the compliance criterion).

\section{Analytical Procedure for Determining the Emission Levels of Selected Monoaromatic Hydrocarbons}

To estimate the emissions of the selected monoaromatic hydrocarbons (BTEX and styrene) and assess the TVOC parameter values associated with the surfaces of the studied small polymeric toys and their plastic packages, the Markes' Micro-Chamber/Thermal Extractor ${ }^{\mathrm{TM}}-\mu-\mathrm{CTE}^{\mathrm{TM}}$ 250 microscale stationary emission chamber system (Markes International Ltd.) was employed. Detailed information about the construction and working parameters of the $\mu$-CTE ${ }^{\mathrm{TM}}$ microscale stationary emission chamber system was presented in previous scientific studies (Marć and Zabiegała 2017; Marć et al. 2015). Before seasoning the chamber, all studied samples of polymeric materials (toys and packages) were removed from the chocolate food product, wiped with a cellulose towel, and weighed so that the emissions of selected chemical compounds from a specific mass of the studied materials could be determined. Due to the fact that the internal volume of the single emission chamber was sufficient to contain whole samples (considering its capacity and loading factor), there was no need to destroy or to reduce the sizes of the studied toys and plastic packages, which provided the opportunity for the same samples to be used in further analyses (FT-IR and the determination of PBDE concentrations).

The two-stage thermal desorption (TD) technique was used for the liberation of analytes from the sorption medium (Tenax TA). The thermal desorption units were attached to the gas chromatography with flame-ionisation detection (GC-FID) and GC-MS systems. Details about the parameters and the conditions used for the liberation, separation and final identification and quantification of analytes are presented in Sect. 1 of Supplementary Data.

\section{Analytical Procedures for Detecting PBDEs}

The surrogate standard ( $1 \mu \mathrm{L}$ of $1 \mathrm{ng} / \mu \mathrm{L}$ F-BDE-47) was added to $0.5 \mathrm{~g}$ of chocolate, and samples were then left for $24 \mathrm{~h}$ to allow them to reach equilibrium. Subsequently, analytes were extracted from the samples twice (for $30 \mathrm{~min}$ and with $3 \mathrm{~mL}$ of $\mathrm{n}$-hexane added at both stages of extraction) by ultrasound-assisted extraction (UAE). Before combining the extracts, they were centrifuged (for $5 \mathrm{~min}$ at $2000 \mathrm{rpm}$ ). The combined extracts were placed in test tubes and evaporated under a gentle stream of nitrogen to a volume of $2 \mathrm{~mL}$. Then, $2 \mathrm{~mL}$ of concentrated sulfuric acid (VI) was added to the extracts and the test tubes were shaken for $1 \mathrm{~min}$ for the mineralisation of interfering compounds and the hydrolysis of fats. Next, the extracts were centrifuged (for $5 \mathrm{~min}$ at $2000 \mathrm{rpm}$ ) to separate the aqueous and organic phases. The upper organic phase was collected with a Pasteur pipette, and then a small dash of anhydrous sodium sulphate was added to remove the remaining water. The extract was centrifuged again (for $5 \mathrm{~min}$ at $2000 \mathrm{rpm}$ ), and upper layer was decanted, and evaporated almost to dryness. The residue was then reconstructed in $100 \mu \mathrm{L}$ of isooctane and the internal standard ( $1 \mu \mathrm{L}$ of $1 \mathrm{ng} / \mu \mathrm{L}$ PCB-209) was added. Three samples were taken and extracted in this way from each chocolate egg.

In the case of the polymeric samples of toys and their packages, a $0.5 \mathrm{~g}$ sample was also used (except for toy samples from product_3, for which $0.2 \mathrm{~g}$ samples were used because the total weights of these toys were smaller), to which the surrogate standard was added $(1 \mu \mathrm{L}$ of $1 \mathrm{ng} / \mu \mathrm{L}$ F-BDE-47) and samples were left to equilibrate for $24 \mathrm{~h}$. Three samples of the polymeric packaging and toy were taken and extracted from each product.

PBDEs were extracted from polymeric samples using UAE. The two 30 min cycles with $3 \mathrm{ml}$ THF as extraction solvent was used to ensure complete dissolution of the polymer samples. The extracts were centrifuged (for $5 \mathrm{~min}$ at $2000 \mathrm{rpm}$ ) and decanted from the residues each time before combining them. The combined extracts were placed in centrifugation tubes, and then $4 \mathrm{~mL}$ of $n$-hexane was added and they were shaken thoroughly by hand. The addition of $n$-hexane caused 
the precipitation of a polymeric pulp, which was removed by centrifugation (for $5 \mathrm{~min}$ at $2000 \mathrm{rpm}$ ) and the subsequent collection of the liquid phase. Extracts were then evaporated in the test tubes almost to dryness. The residue was reconstituted by the addition of $2 \mathrm{~mL}$ of $n$-hexane. Next, approximately $2 \mathrm{~mL}$ of concentrated sulfuric acid (VI) was added and the samples were shaken by hand (for the mineralisation of interfering compounds and, in the case of chocolate samples, the hydrolysis of fats). The extracts were then centrifuged again (for $5 \mathrm{~min}$ at $2000 \mathrm{rpm}$ ) to separate the aqueous and organic phases. The upper organic phase was collected with a Pasteur pipette, and evaporated almost to dryness. The residue was then reconstructed in $100 \mu \mathrm{L}$ of isooctane and the internal standard ( $1 \mu \mathrm{L}$ of $1 \mathrm{ng} / \mu \mathrm{L}$ PCB-209) was added. Three samples of both the toy and their package were taken and extracted in this way from each chocolate egg.

The content of each of the studied PBDEs in each sample was quantified using a gas chromatograph (Agilent 7890A) connected to a quadrupole mass spectrometer (Agilent 5977A) operating in electron capture negative ionisation mode (ECNI) and selected ion monitoring (SIM) mode, and equipped with an autosampler (Agilent 7693). More details about detecting PBDEs can be found in Sect. 2 of Supplementary Data.

\section{Quality Assurance/Quality Control (QA/QC)}

The precise description of the thermal desorption units attached to the GC-FID and GC-MS systems calibration protocol and applied device was enclosed in previous studies (Marć et al. 2014, 2017, 2021).

Regarding PBDEs, component recovery was determined on the basis of extractions and analyses using the surrogate standard. For quantification 5-point calibration curves with different concentration ranges were used. Linearity was determined based on the coefficient of determination $\left(R^{2}\right)$ values for these curves. Analyses of instrument blank samples (pure isooctane) were performed after every 10 samples in the chromatograph sequence. The analysis of the procedural blank was also performed to determine the influence of the analysis background on the results obtained. More details on QA/QC assurance along with the evaluation criteria used can be found in Sect. 3 of the Supplementary Data.

Detailed information about the QA/QC for the VOCs emission studies can be found also in Sect. 3 of the Supplementary Data.

\section{Results and Discussion}

\section{Characteristics of the Studied Polymeric Materials- FT-IR Analysis and Emissions Studies}

The plastic packages placed inside all of the studied chocolate food products were made of using a mixture of polypropylene (PP) and polyethylene (PE). As for the small toy samples, it was noted that the group of toys defined as product_1 were mainly made of the copolymer acrylonitrile butadiene styrene (ABS) covered by a thin layer of atactic PP. The toys from product_2 were generally made of a mixture of polyamide (PA) 6 and PA 6.6, and the toys from product_3 were generally made of atactic PP. The FT-IR spectra of selected small toys from studied group of products and their plastic packages are shown in Fig. 2.

In the FT-IR spectra obtained for the toys defined as product_1 (Fig. 2), characteristic peaks at $3022 \mathrm{~cm}^{-1}$ and $750 \mathrm{~cm}^{-1}$ associated with stretching vibrations of the $\mathrm{C}-\mathrm{H}$ bonds present in the aromatic ring were observed. The absorption maximum detected at the wavenumber of approximately $1600 \mathrm{~cm}^{-1}$ corresponds to the stretching vibration of $\mathrm{C}=\mathrm{C}$ bonds and the bonds present in an aromatic ring (Munteanu and Vasile 2005). The absorption bands found at wavenumbers of approximately 1493 and $1452 \mathrm{~cm}^{-1}$ as well as at 1026, 9604, and $696 \mathrm{~cm}^{-1}$, are characteristic peaks for styrene-butadiene copolymers and their derivatives, respectively. The maximum peaks seen at wavenumbers of approximately 1493, 1452, and $1026 \mathrm{~cm}^{-1}$ are associated with $\mathrm{CH}_{2}$ groups' deformation vibrations in butadiene segments, as well as the stretching vibrations of the carbon atoms in an aromatic ring. An absorption peak detected at the wavenumber of approximately $696 \mathrm{~cm}^{-1}$ might also be associated with the bending vibrations of the $\mathrm{C}-\mathrm{H}$ groups out of the plane of the benzene structure, which is one of the substituent characteristics of styrene copolymers, such as ABS, PS, or styrene-butadiene rubber (SBR) (Motyakin and Schlick 2006; Olmos et al. 2014). Indeed, this observation was the reason why the main polymer that was used to manufacture the studied toys from product_1 was concluded to be the ABS copolymer, as discussed below. Additional data that support this statement are the relative hardness of the studied samples. Compared to the other two groups of polymeric materials, the studied toys in this group (product_1) were very hard to cut or crush into small pieces suitable for use in further steps in the analytical procedure associated with PBDEs analysis. Another aspect of these polymeric toys made of ABS copolymer is the fact that they were covered with a thin film of atactic PP, the presence of which was confirmed by FT-IR analysis. The presence of this thin polymeric layer made it possible to 

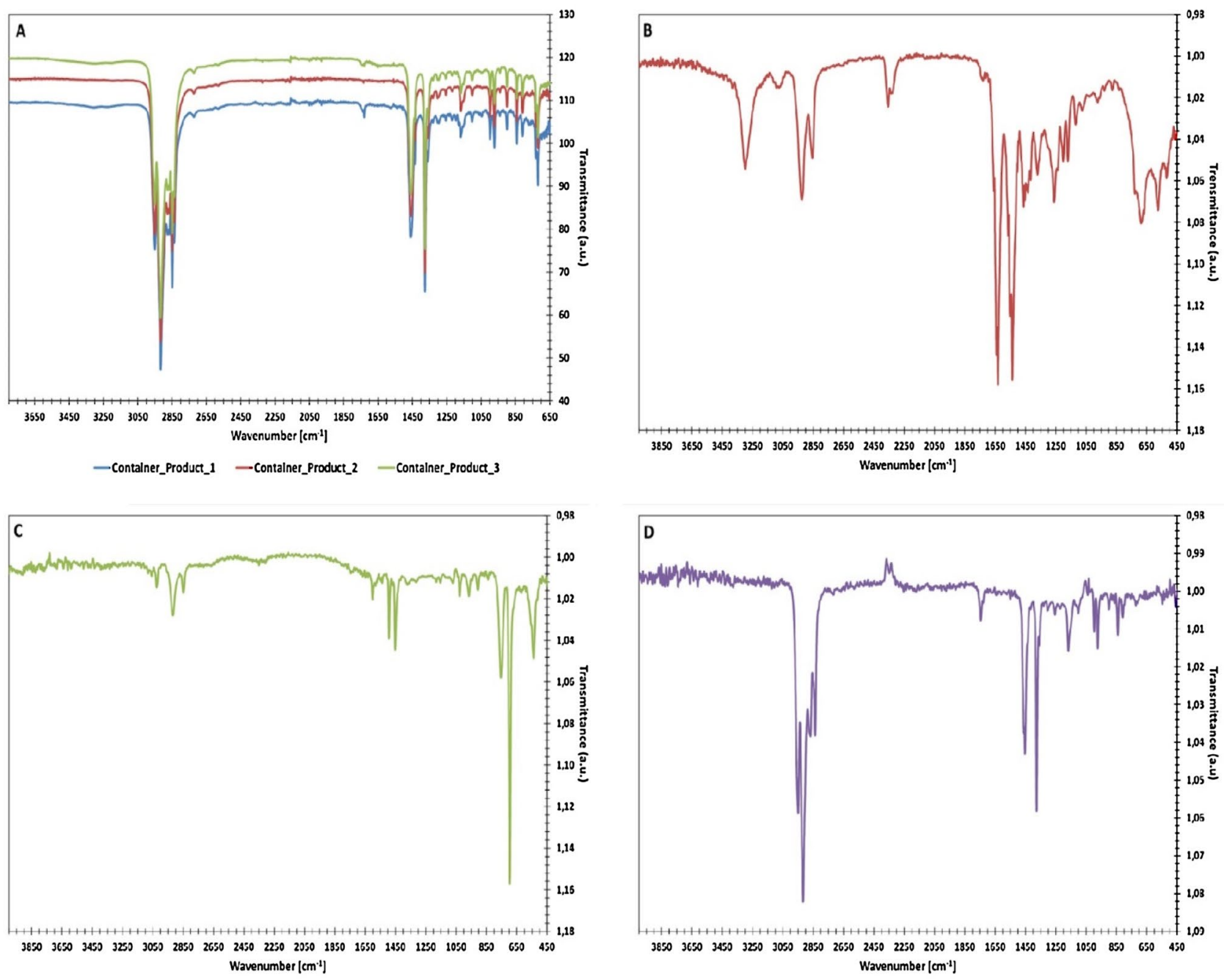

Fig. 2 FT-IR spectra of selected small toys from studied group of products and their plastic packages: A FT-IR spectra of three types of plastic packages; B FT-IR spectra of a products from group no.

1 (product_1); C FT-IR spectra of products from group no. 2 (product_2); D FT-IR spectra of a products from group no. 3 (product_3)

introduce and immobilise the pigment on the surface of the ABS copolymer. For this reason, the FT-IR analysis of the cores of the studied toys confirmed that they were made of ABS copolymer, but the external surfaces of the investigated toys from product_1 were covered by a thin film of atactic PP.

For the small toys from product_2, characteristic absorption bands were observed at wavenumbers 2856 and $2926 \mathrm{~cm}^{-1}$, which might correspond to the stretching vibrations of the $\mathrm{C}-\mathrm{H}$ bonds present in the $-\mathrm{CH}_{2}$ groups of aliphatic chains. The absorption peaks detected at wavenumbers of approximately 3230 and $3076 \mathrm{~cm}^{-1}$ might correspond to the stretching vibrations of the $\mathrm{N}-\mathrm{H}$ group. The absorption peak seen at the wavenumber of approximately $1632 \mathrm{~cm}^{-1}$ is characteristic of the vibration of the carbonyl group, $-\mathrm{C}=\mathrm{O}$. The absorption band found at approximately $1537 \mathrm{~cm}^{-1}$ is associated with the crystalline phases of an

un-associated amide (Porubská et al. 2012). The absorption band detected in the range $1416-1461 \mathrm{~cm}^{-1}$ might be characteristic for scissoring vibrations in trans-conformation of the $-\mathrm{CH}_{2}$ group adjacent to $\mathrm{N}-\mathrm{H}$ and $\mathrm{C}=\mathrm{O}$ groups (Wu et al. 2004; Jeziórska et al. 2011). Taking the above information into account, it could be concluded that the studied toys from product_2 were mainly made of a mixture of PA 6 and PA 6.6.

The FT-IR spectra of the last group of small toys studied (product_3) contained a characteristic absorption band over the range $2949-2837 \mathrm{~cm}^{-1}$, which might correspond to the symmetric and asymmetric stretching vibrations of the $\mathrm{C}-\mathrm{H}$ bonds present in the $-\mathrm{CH}_{2}$ groups of aliphatic chains. The absorption band detected in the range $1452-1458 \mathrm{~cm}^{-1}$ is characteristic for the asymmetric bending vibrations of the $-\mathrm{CH}_{2}$ and $-\mathrm{CH}_{3}$ groups (Ebadi-Dehaghani et al. 2016). The peak observed at approximately $1375 \mathrm{~cm}^{-1}$ corresponds to 
the stretching vibrations of $\mathrm{C}=\mathrm{C}$ bonds (Nekhoroshev et al. 2005). In the range from 840 to $1165 \mathrm{~cm}^{-1}$, several absorption peaks were seen that may correspond to various types of vibrations, including the bending, stretching, and rocking of $-\mathrm{CH}_{2}$ and $-\mathrm{CH}_{3}$ groups (Santhoskumar et al. 2010). Considering the abovementioned data, it could be concluded that the toys from product_3 were generally made of atactic PP.

\section{BTEX, Styrene and TVOCs Emission Studies}

As for the measured emissions of selected monoaromatic hydrocarbons from containers made of PE, it should be noted that these packaging samples were open during the seasoning step in the emission chamber. For this reason, it was observed (see the Sect. 4 in Supplementary Data) that there were significant variabilities in their hydrocarbon emissions. These variations might have been caused by the different types of toys placed inside the container used for $\mathrm{PE}$ analysis. It might be concluded that PE-based packages might adsorb chemical compounds emitted from the small plastic toys placed inside them on their inner-surfaces. This is highly probable because of the long-term exposure of packages to toy emissions, the porous internal surface of the packaging, and the package's polymeric structure. Because of this, two assumptions can be made about PE-based packages: (i) they represent an additional source of emissions of chemicals that might migrate direct from PE impurities and polymers because of the thermal degradation of PE; and (ii) they can be considered a specific type of sorption material and/or membrane that controls the transportation of chemicals emitted from the small toy placed inside the PE-based container in the gaseous phase, and then directly to the chocolate.

A clear relationship between the main type of polymer used in each type of sample and the emissions of specific monoaromatic hydrocarbons therefrom was observed. For small toys mainly made of ABS, ethylbenzene and styrene were characterised by the most intensive emission from these products. The emissions of ethylbenzene and styrene ranged from 16.81 to $33.92 \mathrm{ng} / \mathrm{g}$ and from 0.64 to $5.86 \mathrm{ng} / \mathrm{g}$, respectively. The emission of these monoaromatic hydrocarbons is mainly associated with the solvent residues and reaction substrates used in the polymerisation process producing ABS. Ethylbenzene is commonly employed to synthesise styrene, and styrene is one of the main elements used during ABS polymerisation. For this reason, the increased emission of these two monoaromatic hydrocarbons from the studied polymeric materials provides information about the main type of polymer (in the above case, ABS) that was used during the manufacturing process of the studied plastic products.

For product_2, the small toys therein were mainly made of PA according to the results of FT-IR analysis. PA small toys exhibited medium emission levels of toluene, ethylbenzene, and p,m-xylene. The emissions of these monoaromatic hydrocarbons ranged from 0.52 to $2.26 \mathrm{ng} / \mathrm{g}$, from 0.54 to $1.31 \mathrm{ng} / \mathrm{g}$, and from 2.24 to $4.87 \mathrm{ng} / \mathrm{g}$, respectively. Furthermore, in comparison to the other small plastic toys studied, the toys made of PA were characterised by the lowest values of the TVOC parameter (range: from 35.50 to $103.01 \mathrm{ng} / \mathrm{g}$ ). This might lead to the conclusion that materials made of PA are the source of the least intense monoaromatic hydrocarbon emissions, as well as emissions of different types of aliphatic hydrocarbons.

As for PP small toys, these were characterised as exhibiting the highest emissions of ethylbenzene and $\mathrm{p}, \mathrm{m}$-xylene, specifically ranging from 4.17 to $8.29 \mathrm{ng} / \mathrm{g}$ and from 0.90 to $5.89 \mathrm{ng} / \mathrm{g}$, respectively. The relatively high content of monoaromatic hydrocarbons in and their consequent emissions from PP-based materials might be caused by the residues of solvents applied during the final manufacturing process of these products. Additionally, the PP-based toys were characterised as having the highest values of the TVOC parameter (range from 2321.84 to $3023.37 \mathrm{ng} / \mathrm{g}$ ). This might confirm that plastic materials made of PP are sources of intense emissions of chemical compounds, including monoaromatic hydrocarbons, and probably also aliphatic hydrocarbons, as a result of thermal degradation of PP. This could also lead to other interesting phenomena involving linear polymers like PE and PP, including the fact that they are characterised as giving off intense emissions of aliphatic hydrocarbons (which has a strong impact on the value of the TVOC parameter) and thus might be considered one of the main sources of the emission of the abovementioned compounds.

As for the emissions of selected VOCs from PE containers, it should be highlighted that these samples were opened during the seasoning step in the emission chamber. For this reason, it was observed that there were significant variabilities in their hydrocarbon emissions. These variations might have been caused by the different types of toys placed inside the container used for PE analysis. In cases when the small plastic toy placed in the chamber was made of PP or PA, the emissions of monoaromatic hydrocarbons measured were only higher than $2 \mathrm{ng} / \mathrm{g}$ once, in the case of toluene $(2.52 \mathrm{ng} / \mathrm{g})$ emitted from the PE-based container in which a PA-based small toy was placed. In cases when the small toy was made of ABS and was earlier placed inside a PE-based package, the emissions of monoaromatic hydrocarbons from the PE container were much higher in comparison to those measured under the previously mentioned circumstances. For PE-based packages in which the small toy was mainly made of ABS, the most intensive emissions detected were those of ethylbenzene and styrene. The emissions of ethylbenzene and styrene ranged from 4.91 to $187.33 \mathrm{ng} / \mathrm{g}$ and from 0.43 to $26.80 \mathrm{ng} / \mathrm{g}$, respectively. 


\section{PBDE Concentrations}

Based on the results obtained (Table 2), it can be concluded that very different concentrations of PBDEs can occur in chocolate egg products, depending on the price of the product and the type of polymer of which the toy therein is made. No PBDEs were detected in any of the components of the most expensive, high-quality original product (product_1), wherein the toy was made of ABS covered by a thin layer of atactic polypropylene. In chocolate and package samples of product_2, PBDE levels were below the LOD, with the exception of one toy made of polyamide, in which the concentrations of most representative PBDEs were below the LOQ except BDE-99 and BDE-154, whose concentration were quantified (see Table 2). These two PBDEs are amongst the dominant compounds that are present in the commercial mixture c-pentaBDE. The cheapest product (product_3) contained a wide range of PBDEs, except for samples of its packaging, wherein only BDE-99 was detected at low concentrations $(0.00120 \pm 0.00011 \mathrm{ng} / \mathrm{g})$. This shows that, in general, the packaging used in this product meets the requirements for polymeric materials intended for use in direct contact with food. Overall, higher concentrations of PBDEs were detected in the chocolate of product_3 than in its toys (made of atactic polypropylene), except for BDE-28 and BDE-154. This may indicate there is a high potential for the migration and accumulation of PBDEs in the chocolate of this product. It can also be observed that for toys where no PBDEs were detected, the content in chocolate samples was also below MQLs-this may be an argument that the

Table 2 Concentrations of PBDEs in the parts of studied commercially available child-dedicated food products (ng/g)

\begin{tabular}{|c|c|c|c|c|c|c|c|c|c|c|}
\hline \multicolumn{3}{|c|}{ Sample type } & \multirow{2}{*}{$\frac{\text { BDE-28 }}{<\mathrm{LOD}}$} & \multirow{2}{*}{$\frac{\text { BDE-47 }}{<\text { LOD }}$} & \multirow{2}{*}{$\frac{\text { BDE-100 }}{<\text { LOD }}$} & \multirow{2}{*}{$\frac{\text { BDE-99 }}{<\text { LOD }}$} & \multirow{2}{*}{$\frac{\text { BDE-154 }}{<\text { LOD }}$} & \multirow{2}{*}{$\frac{\text { BDE-153 }}{<\text { LOD }}$} & \multirow{2}{*}{$\frac{\text { BDE-183 }}{<\text { LOD }}$} & \multirow{2}{*}{$\frac{\text { BDE-209 }}{<\text { LOD }}$} \\
\hline Product_1 & $\begin{array}{l}\text { Chocolate } \\
n=8\end{array}$ & Mean (ng/g) & & & & & & & & \\
\hline & $\begin{array}{l}\text { Package } \\
n=8\end{array}$ & Mean (ng/g) & $<\mathrm{LOD}$ & $<\mathrm{LOD}$ & $<\mathrm{LOD}$ & $<$ LOD & $<\mathrm{LOD}$ & $<\mathrm{LOD}$ & $<\mathrm{LOD}$ & $<\mathrm{LOD}$ \\
\hline & $\begin{array}{l}\text { Toy } \\
n=8\end{array}$ & Mean (ng/g) & $<\mathrm{LOD}$ & $<\mathrm{LOD}$ & $<\mathrm{LOD}$ & $<$ LOD & $<\mathrm{LOD}$ & $<\mathrm{LOD}$ & $<\mathrm{LOD}$ & $<\mathrm{LOD}$ \\
\hline \multirow[t]{8}{*}{ Product_2 } & $\begin{array}{l}\text { Chocolate } \\
n=8\end{array}$ & Mean (ng/g) & $<\mathrm{LOD}$ & $<\mathrm{LOD}$ & $<\mathrm{LOD}$ & $<$ LOD & $<\mathrm{LOD}$ & $<\mathrm{LOD}$ & $<\mathrm{LOD}$ & $<\mathrm{LOD}$ \\
\hline & $\begin{array}{l}\text { Package } \\
n=8\end{array}$ & Mean (ng/g) & $<\mathrm{LOD}$ & $<\mathrm{LOD}$ & $<\mathrm{LOD}$ & $<\mathrm{LOD}$ & $<\mathrm{LOD}$ & $<\mathrm{LOD}$ & $<\mathrm{LOD}$ & $<\mathrm{LOD}$ \\
\hline & \multirow{6}{*}{$\begin{array}{l}\text { Toy } \\
n=8\end{array}$} & Mean (ng/g) & $<\mathrm{LOD}$ & $<\mathrm{LOQ}$ & $<\mathrm{LOQ}$ & 0.83 & 0.208 & $<$ LOD & $<\mathrm{LOD}$ & $<$ LOD \\
\hline & & Median (ng/g) & - & - & - & 0.79 & 0.200 & - & - & - \\
\hline & & Std (ng/g) & - & - & - & 0.23 & 0.019 & - & - & - \\
\hline & & $\% \mathrm{CV}$ & - & - & - & 28 & 9.2 & - & - & - \\
\hline & & $\operatorname{Min}(n g / g)$ & - & - & - & 0.44 & 0.194 & - & - & - \\
\hline & & $\operatorname{Max}(n g / g)$ & - & - & - & 1.5 & 0.230 & - & - & - \\
\hline \multirow[t]{18}{*}{ Product_3 } & \multirow{6}{*}{$\begin{array}{l}\text { Chocolate } \\
n=8\end{array}$} & Mean (ng/g) & $<\mathrm{LOQ}$ & 13.4 & 5.32 & 13.5 & $<\mathrm{LOQ}$ & 14.4 & 5.8 & 1768 \\
\hline & & Median (ng/g) & - & 13.1 & 5.10 & 13.7 & - & 18.6 & 5.5 & 1718 \\
\hline & & Std (ng/g) & - & 2.0 & 0.79 & 2.2 & - & 6.9 & 1.1 & 289 \\
\hline & & $\% \mathrm{CV}$ & - & 15 & 15 & 16 & - & 48 & 19 & 16 \\
\hline & & Min (ng/g) & - & 10.8 & 3.93 & 8.4 & - & 5.7 & 4.8 & 1391 \\
\hline & & Max (ng/g) & - & 19.0 & 6.78 & 17.5 & - & 25.7 & 7.7 & 2388 \\
\hline & \multirow{6}{*}{$\begin{array}{l}\text { Package } \\
n=8\end{array}$} & Mean (ng/g) & $<\mathrm{LOQ}$ & $<$ LOQ & $<\mathrm{LOQ}$ & 0.00120 & $<\mathrm{LOQ}$ & $<$ LOD & $<\mathrm{LOQ}$ & $<\mathrm{LOD}$ \\
\hline & & Median (ng/g) & - & - & - & 0.00124 & - & - & - & - \\
\hline & & Std (ng/g) & - & - & - & 0.00011 & - & - & - & - \\
\hline & & $\% \mathrm{CV}$ & - & - & - & 8.8 & - & - & - & - \\
\hline & & $\min (n g / g)$ & - & - & - & 0.00124 & - & - & - & - \\
\hline & & $\max (n g / g)$ & - & - & - & 0.00124 & - & - & - & - \\
\hline & \multirow{6}{*}{$\begin{array}{l}\text { Toy } \\
n=8\end{array}$} & mean(ng/g) & 2.00 & 1.87 & 0.452 & 2.27 & 0.62 & 0.56 & 1.08 & 633 \\
\hline & & median (ng/g) & 2.01 & 1.35 & 0.417 & 2.38 & 0.56 & 0.55 & 1.00 & 732 \\
\hline & & std (ng/g) & 0.50 & 0.72 & 0.075 & 0.86 & 0.15 & 0.19 & 0.41 & 204 \\
\hline & & $\% \mathrm{CV}$ & 25 & 38 & 17 & 38 & 24 & 34 & 38 & 32 \\
\hline & & $\min (n g / g)$ & 0.42 & 0.45 & 0.379 & 0.49 & 0.43 & 0.19 & 0.28 & 45 \\
\hline & & $\max (n g / g)$ & 5.60 & 4.63 & 0.590 & 3.87 & 0.92 & 1.12 & 3.23 & 1230 \\
\hline
\end{tabular}


main source of PBDEs in chocolate is migration from the toy. PBDEs migration can be further justified by the fact that chocolate is rich in fat, to which PBDEs have a high affinity. However, due to the practical absence of PBDEs in toy packaging, the most likely hypothesis seems to be that the toys are contaminated by the waste stream and the high presence of these xenobiotics in the chocolate is due to another source, e.g. milk, butter/oil, the production process (Pietron et al. 2021; Zacs et al. 2021; Konstantas et al. 2018).

Furthermore, it can be observed that the higher concentration of PBDEs in the cheapest product (product_3) corresponds to an increased emission profile of TVOCs. In the case of the toy from product_3, the TVOC emissions were more than 3 times higher than those of the toy from product_1, in which PBDEs were present below the LOD level. This indicates there is an increased exposure of children to various types of chemical compounds from the tested product_3, despite the presence of the CE declaration of conformity on this product. However, it should be mentioned that according to the EU Directive on the Restriction of Hazardous Substances (Directive 2015/863/EC 2015) the maximum concentrations of PBDEs permitted to be included in the homogeneous material of articles intended for daily use should be at $1000 \mathrm{ppm}$. Our results do not exceed this requirement.

The presence of PBDE in polymer toys can only be explained by probable existence of incorrect recycling practices for polymers containing these pollutants. At the end-oflife of the consumer products, many polymeric materials are recycled and the additives they contain, such as flame retardants and plasticizers, may be unintentionally transferred to the newly manufactured goods (Guzzonato et al. 2017). Recycled materials are likely to be an important source of these additives in toys and therefore their reuse in products intended for children should be subject to stricter controls. Incorrect recycling practices lead to non-compliance with the CE certificate and cause the re-introduction of phasedout PBDEs into turnover (Shittu et al. 2021). From a health protection point of view, the question is how many unexplored paths of unexpected exposure to PBDEs can exist (Abbasi et al. 2019). This issue is still relevant and needs to be further explored, despite the ban on using PBDEs in industrial production (Sharkey et al. 2020). In addition, consideration should be given to the balance between the effort required to recycle polymers and the benefits of preventing their re-introduction into the environment. Available tools for determining bromine compounds, such as X-ray fluorescence (XRF), allow rapid selection of a given polymer due to the probability of the presence of brominated flame retardants, including PBDEs (UNEP 2017). Nevertheless, recycling undoubtedly involves additional financial costs, requires advanced technological solutions and can take considerable time (Shittu et al. 2021). With regard to environmental safety, good recycling procedures for polymers, including separation of PBDEs and other hazardous substances, play an important role. Most likely, recycled materials are an important source of PBDEs in toys and therefore, their (re)use in products for children should be subject to stricter restrictions.

The data we obtained were compared in Supplementary Data Table S9 with other studies on PBDE content in foods (especially milk) and polymeric toys. Based on the comparison of our results of PBDE determination in chocolate samples with literature data on PBDE content in milk samples, it can be concluded that the analyte content determined by us is many times higher than the data reported for milk samples. It should be emphasized that the reports for milk samples refer to the concentration per mass of fat contained in milk. In our case the PBDE content was reported for whole chocolate samples. However, even after estimating the presence of PBDEs in the fat contained in the chocolate itself (the manufacturer reported a fat content of $31.7 \%$ for product_3), we can conclude that the PBDEs we found are still alarmingly high compared to literature reports for milk samples. It is also worth mentioning at this point that the Scientific Committee of the Federal Agency for the Safety of the Food Chain has proposed limits chemical contaminants (flame retardants, perfluoralkyl substances, dioxins and dioxin-like PCB and benzene) in foodstuffs). These data enable an assessment of the risk for customers' health. However, it should be emphasized that in the mentioned document the data about proposed action limit for the sum of PBDE refer to the content per mass of fat present in different types of food. In our study we did not make such a distinction and made the determination per total weight of chocolate. Therefore, we will not attempt to estimate the concentration of PBDEs in the fat mass of chocolate alone. The fact is that our results are alarming-regardless of whether they refer to concentrations in the fat alone or in the whole product (FASFC, Advice 15-2017).

In the case of comparing our results obtained for polymer toys with the results published on PBDE content in toys and children's play mats, it can be concluded that our results are comparable with those obtained by other researchers. However, it is noticeable that the concentration of BDE-209 is much higher in the samples of polymeric toys of product_3, which was the object of our study.

\section{Forward-Looking Considerations: Estimation of Children's Exposure Risk to PBDEs}

According to the best of our knowledge, the described study was performed first time for PBDEs in this type of consumer product (components of chocolate egg with the toy placed inside). For this reason, in order to expand this issue, 
predictive considerations were made by theoretically estimating the exposure of children in contact with the test product. All available routes from some of the products studied, i.e., the chocolate egg (by ingestion) and the polymer toy or packaging (by inhalation, dermal contact, and oral ingestion), were considered. Section 6 of the Supplementary Data presents the workflow leading to the calculation of exposure by these exposure routes. For compounds that were not detected in the chocolate samples (BDE-28 and BDE-154), LOQ values were used to estimate children's exposure-as the upper bound of the set of PBDE concentration results (Mathworld 2021).

The estimated risk of children being exposed to PBDEs as a result of contact with different parts of the studied commercially available chocolate food product_3 is presented in Table 3.

The RfD values for BDE-47, $-99,-153$, and -209 were not exceeded in any type of sample (see Table S8). The chemical compound to which the exposure of children is the highest via the studied products is BDE-209 because the highest concentrations of this compound were observed in the chocolate and toy components of these products. Much higher exposure risks were estimated for the youngest age group (3-5 years) than for other subgroups, which is mainly due to the lower body weights of these age groups, as well as them exhibiting much more frequent hand-to-mouth behaviour. The predominant route of exposure is via the oral route, especially chocolate ingestion.

Additionally, for oral exposure (i.e., both hand-to-mouth and chocolate ingestion), the hazard quotient (HQ) and cancer risk associated with the intake of BDE-209 ( $\left.\mathrm{CR}_{\mathrm{BDE}-209}\right)$ were estimated. The method for estimating these parameters can be found in Sect. 6 of Supplementary Data. Hazard quotients (HQs) calculated using oral exposure values (chocolate ingestion and hand-to-mouth ingestion) indicated that there is no significant risk associated with PBDE exposure from the tested product_3 (all HQ $<1$ ). However, it should be noted that these data were calculated based on the case where a child only eats one chocolate egg per day. Considering that eating chocolate was found to be the dominant route of exposure to PBDEs in the studied food products, it was concluded that adverse effects are expected for the 3-5 years old age group when three or more chocolate eggs are consumed per day. The detailed HQ values calculated herein are included in Table 4. There are also data on HQ values for food products obtained in other studies. Our estimates indicate higher HQ values for individual PBDEs compared to literature data for foods of various types. However, it should be emphasized that our estimated values refer to the exposure of children in different age groups, whereas the literature data consider adult exposure. Children belong to a particularly sensitive group in terms of health effects from exposure to PBDEs. Hence, the estimation of HQ values as

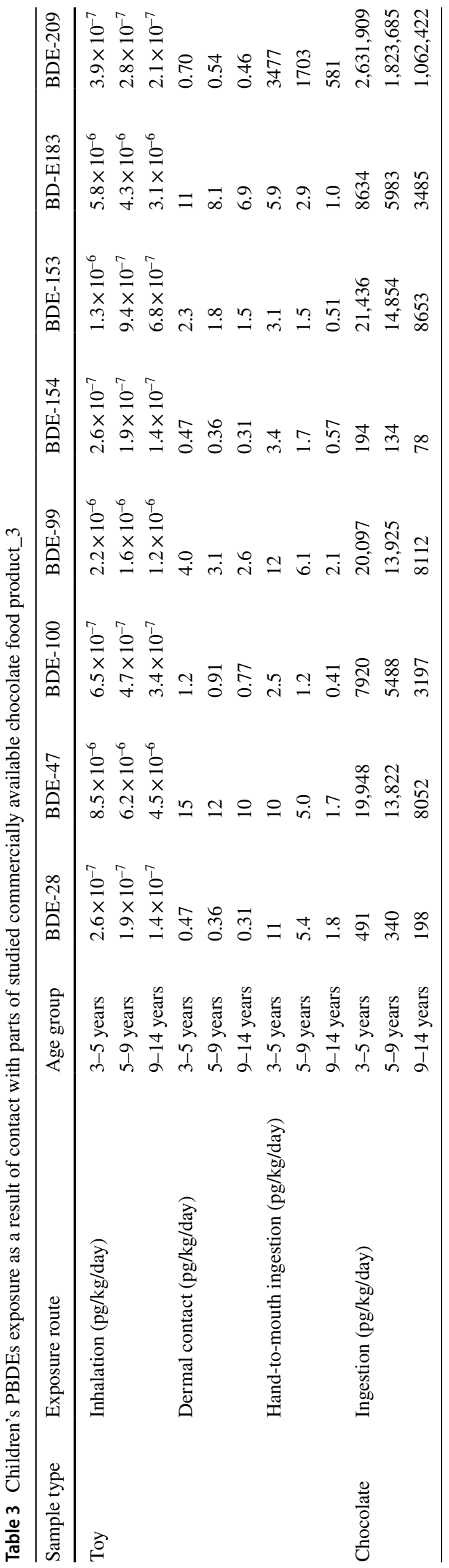


Table 4 The hazard quotients (HQs) and cancer risk $\left(\mathrm{CR}_{\mathrm{BDE}-209}\right)$ of children's ingestion exposure to PBDEs via toy and chocolate of product_3

\begin{tabular}{|c|c|c|c|c|c|}
\hline Age group & $\mathrm{HQ}_{\mathrm{BDE}-47}$ & $\mathrm{HQ}_{\mathrm{BDE}-99}$ & $\mathrm{HQ}_{\mathrm{BDE}-153}$ & $\mathrm{HQ}_{\mathrm{BDE}-209}$ & $\mathrm{CR}_{\mathrm{BDE}-209}$ \\
\hline $3-5$ years & 0.20 & 0.20 & 0.11 & 0.38 & $3.7 \times 10^{-10}$ \\
\hline 5-9 years & 0.14 & 0.14 & 0.074 & 0.26 & $3.8 \times 10^{-10}$ \\
\hline $9-14$ years & 0.081 & 0.081 & 0.043 & 0.15 & $2.5 \times 10^{-10}$ \\
\hline Sample type & $\mathrm{HQ}_{\mathrm{BDE}-47}$ & $\mathrm{HQ}_{\mathrm{BDE}-99}$ & $\mathrm{HQ}_{\mathrm{BDE}-153}$ & $\mathrm{HQ}_{\mathrm{BDE}-209}$ & Refs. \\
\hline \multicolumn{6}{|l|}{ Literature data } \\
\hline Meat & $2.79 \times 10^{-4}$ & $1.53 \times 10^{-4}$ & $8.27 \times 10^{-6}$ & $5.76 \times 10^{-5}$ & Martellini et al. (2016) \\
\hline Dairy products & $3.02 \times 10^{-3}$ & $1.50 \times 10^{-3}$ & $5.41 \times 10^{-5}$ & $8.54 \times 10^{-4}$ & Martellini et al. (2016) \\
\hline Eggs & $9.43 \times 10^{-5}$ & $5.78 \times 10^{-5}$ & $5.23 \times 10^{-6}$ & $2.32 \times 10^{-5}$ & Martellini et al. (2016) \\
\hline Fish and mussels & $1.29 \times 10^{-3}$ & $1.74 \times 10^{-4}$ & $2.37 \times 10^{-5}$ & $2.70 \times 10^{-5}$ & Martellini et al. (2016) \\
\hline
\end{tabular}

a result of children's exposure to PBDEs in contact with a food product that is dedicated to children is an important contribution to the knowledge of the prognosis resulting from such exposure.

The calculation of cancer risk (for BDE-209 only) values showed that the oral exposure to PBDEs through the different parts of the commercially available child-dedicated food products analysed in this study can be classified as 'low risk'.

According to our results, a comparison of potential sources of PBDEs presence in chocolate leads to the conclusion that, from the point of view of children's exposure, contamination of PBDEs in the food chain is more significant than re-introduction due to recycling. Considering that dietary pathway are the dominant route of exposure to PBDEs, direct consumption of contaminated food can have more significant health effects. On the other hand, inadequate polymer recycling practices contribute to the re-introduction of PBDEs into the environment, which can also result in soil and air pollution, and consequently lead to the occurrence of PBDEs, e.g. in meat from farm animals, milk and dairy products (Pietron et al. 2021; Zacs et al. 2021; Konstantas et al. 2018). Consequently, in this context, inappropriate recycling procedures may take on much greater importance in the environmental fate of the PBDEs.

\section{Conclusions}

The combination of FT-IR analysis with measurements of the monoaromatic hydrocarbon emissions from polymeric materials provides valuable information about the potential solvents and monomer residues that might occur in their plastic materials and be emitted directly via the gaseous phase and further migrate into the chocolate food product. Furthermore, the cover/thin film of polymer applied to several types of toy samples may be an additional source of hydrocarbon emissions, but might also be considered a special type of membrane that hinders the emission of chemical compounds that occur in the main polymeric materials (the core of the toy).

Our analytical procedures can serve as an alternative to the standard procedure for polymeric analysis, which uses a cryogenic mill to grind polymers. This will allow the costs associated with the use of liquid nitrogen to be avoided, whilst maintaining a similar sample preparation time.

PBDEs were detected in the different parts of commercially available child-dedicated chocolate food products depending on the type (origin) and price of the products. This demonstrates the need for consumer knowledge on the safety of such products available on the Polish market to be broadened. This is one of the many examples of studies showing that products sold on the European market do not always comply with the CE declaration of conformity.

The low-cost products tested were characterised by the presence of all eight PBDE analytes. The highest PBDE concentrations, which ranged from $5.32 \pm 0.79$ to $1768 \pm 289 \mathrm{ng} / \mathrm{g}$, were detected in chocolate samples. In the more expensive original products tested, the PBDE levels were found to be below the LOD.

Despite the detection of PBDEs in all parts of some of the tested food products intended for children, no high risk associated with exposure of children to PBDEs was found, assuming that a child eats only one chocolate egg per day.

In the future, this type of research should be extended to include analysis of the air inside the toy packaging and between the packaging and chocolate, and should also assess the presence of other harmful SVOCs in these products. Another important future task is to develop a procedure to estimate the potential for PBDEs to migrate from a polymeric toy to chocolate by diffusion.

Supplementary Information The online version contains supplementary material available at https://doi.org/10.1007/s12403-021-00428-2. 
Acknowledgements The authors would like to thank Krzysztof Formela and Marta Przybysz-Romatowska from the Department of Polymer Technology, GUT, for preforming the FT-IR analysis.

Funding Funding was provided by National Science Centre, Poland under the Preludium16 programme in the years 2019-2022, project no. 2018/31/N/ST10/03664.

Data Availability All data support our published claims and comply with field standards.

Code Availability Not applicable.

\section{Declarations}

Conflict of interest The authors have no conflict of interest.

Open Access This article is licensed under a Creative Commons Attribution 4.0 International License, which permits use, sharing, adaptation, distribution and reproduction in any medium or format, as long as you give appropriate credit to the original author(s) and the source, provide a link to the Creative Commons licence, and indicate if changes were made. The images or other third party material in this article are included in the article's Creative Commons licence, unless indicated otherwise in a credit line to the material. If material is not included in the article's Creative Commons licence and your intended use is not permitted by statutory regulation or exceeds the permitted use, you will need to obtain permission directly from the copyright holder. To view a copy of this licence, visit http://creativecommons.org/licenses/by/4.0/.

\section{References}

Abbasi G, Li L, Breivik K (2019) Global historical stocks and emissions of PBDEs. Environ Sci Technol 53:6330-6340

Azwa ZN, Yousif BF, Manalo AC, Karunasena W (2013) A review on the degradability of polymeric composites based on natural fibres. Mater Des 47:424-442

Bauer A, Jesus F, Gomez Ramos MJ, Lozano A, Fernandez-Alba AR (2019) Identification of unexpected chemical contaminants in baby food coming from plastic packaging migration by high resolution accurate mass spectrometry. Food Chem 295:274-288

Bhaskar T, Hall WJ, Mitan NMM, Muto A, Williams PT, Sakata Y (2007) Controlled pyrolysis of polyethylene/polypropylene/polystyrene mixed plastics with high impact polystyrene containing flame retardant: effect of decabromo diphenylethane (DDE). Polym Degrad Stabil 92(2):211-221

Cowell WJ, Margolis A, Rauh VA, Sjoedin A, Jones R, Wang Y, Garcia W, Pereira F, Wang S, Herbstman JB (2018) Associations between prenatal and childhood PBDE exposure and early adolescent visual, verbal and working memory. Environ Int 118:9-16

Currier HA, Fremlin KM, Elliott JE, Drouillard KG, Williams TD (2020) Bioaccumulation and biomagnification of PBDEs in a terrestrial food chain at an urban landfill. Chemosphere 238:124577

Directive 2009/48/CE (2009) On safety of and toys. Off J Eur Communities L170:1-37

Directive 2015/863/EC (2015) Restriction of the use of certain hazardous substances (RoHS) in electrical and electronic equipment

Drobna B, Fabisikova A, Conka K, Gago F, Oravcova P, Wimmerova S, Oktapodas Feiler M, Sovcikova E (2019) PBDE serum concentration and preschool maturity of children from Slovakia. Chemosphere 233:387-395
Ebadi-Dehaghani H, Barikani M, Borhani S, Bolvardi B, Khonakdar HA, Jafari SH, Aarabi A (2016) Biodegradation and hydrolysis studies on polypropylene/polylactide/organo-clay nanocomposites. Polym Bull 73:3287-3304

European Union (2011) Commission regulation No. 10/2011 on plastic materials and articles intended to come into contact with food. Off J Eur Union 12:1-89

Fabelova L, Loffredo CA, Klanova J, Hilscherova K, Horvat M, Tihanyi J, Richterova D, Palkovicova Murinova L, Wimmerova S, Sisto R, Moleti A, Trnovec T (2019) Environmental ototoxicants, a potential new class of chemical stressors. Environ Res 171:378-394

FASFC, Advice 15-2017. Scientific Committee of the Federal Agency for the Safety of the Food Chain in regard to action levels for chemical contaminants (flame retardants, perfluoralkyl substances, dioxins and dioxin-like PCB and benzene) in foodstuffs)

Formela K, Marć M, Wang S, Saeb MR (2017) Interrelationship between total volatile organic compounds emissions, structure and properties of natural rubber/polycaprolactone bio-blends crosslinked with peroxides. Polym Test 60:405-412

Guzzonato A, Puype F, Harrad SJ (2017) Evidence of bad recycling practices: BFRs in children's toys and food-contact articles. Environ Sci Process Impacts 19(7):956-963

Hankett JM, Collin WR, Chen Z (2013) Molecular structural changes of plasticized PVC after UV light exposure. J Phys Chem B 117:16336-16344

Harley KG, Rauch SA, Chevrier J, Kogut K, Parra KL, Trujillo C, Lustig RH, Greenspan LC, Sjoedin A, Bradman A, Eskenazi B (2017) Association of prenatal and childhood PBDE exposure with timing of buberty in boys and girls. Environ Int 100:132-138

Hoffman K, Lorenzo A, Butt CM, Hammel SC, Henderson BB, Roman SA, Scheri RP, Stapleton HM, Sosa JA (2017) Exposure to flame retardant chemicals and occurrence and severity of papillary thyroid cancer: a case-control study. Environ Int 107:235-242

Ionas AC, Dirtu AC, Anthonissen T, Neels H, Covaci A (2014) Downsides of the recycling process: harmful organic chemicals in children's toys. Environ Int 65:54-62

Jeziórska R, Świerz-Motysia B, Szadkowska A, Marciniec B, Maciejewski H, Dutkiewicz M, Leszczyńska I (2011) Effect of POSS on morphology, thermal and mechanical properties of polyamide 6. Polimery 56:809-816

Ji H, Liang H, Wang Z, Miao M, Wang X, Zhang X, Wen S, Chen A, Sun X, Yuan W (2019) Associations of prenatal exposures to low levels of polybrominated diphenyl ether (PBDE) with thyroid hormones in cord plasma and neurobehavioral development in children at 2 and 4 years. Environ Int 131:105010

Kelly BC, Ikonomou MG, Blair JD, Gobas FAPC (2008) Bioaccumulation behaviour of polybrominated diphenyl ethers (PBDEs) in a Canadian Arctic marine food web. Sci Total Environ 401(1-3):60-72

Konstantas A, Jeswani HK, Stamford L, Azapagic A (2018) Environmental impact of chocolate production and consumption in the UK. Food Res Int 106:1012-1025

Koren G, Carnevale A, Ling J, Ozsarfati J, Kapur B, Bagli D (2019) Fetal exposure to polybrominated diphenyl ethers and the risk of hypospadias: focus on the congeners involved. J Pediatr Urol 15(4):e1-e6

Kozai T, Kaneko T, Hirai N, Ohki Y (2018) Identification of antioxidants in polymeric insulating materials by tetrahertz absorption spectroscopy. Polym Degrad Stabil 147:284-290

Lattuati-Derieux A, Egasse C, Thao-Heu S, Balcar N, Barabant G, Lavédrine B (2013) What do plastics emit? HS-SPME-GC/MS analyses of new standard plastics and plastic objects in museum collections. J Cult Herit 14:238-247 
Marć M, Zabiegała B (2017) An investigation of selected monoaromatic hydrocarbons released from the surface of polystyrene lids used in coffee-to-go cups. Microchem J 133:496-505

Marć M, Zabiegała B, Namieśnik J (2014) Application of passive sampling technique in monitoring research on quality of atmospheric air in the area of Tczew, Poland. Int J Environ Anal Chem 94:151-167

Marć M, Formela K, Klein M, Namieśnik J, Zabiegała B (2015) The emissions of monoaromatic hydrocarbons from small polymeric toys placed in chocolate food products. Sci Total Environ 530-531:290-296

Marć M, Namieśnik J, Zabiegała B (2017) The miniaturised emission chamber system and home-made passive flux sampler studies of monoaromatic hydrocarbons emissions from selected commercially-available floor coverings. Build Environ 123:1-13

Marć M, Tsakovski S, Tobiszewski M (2021) Emissions and toxic units of solvent, monomer and additive residues released to gaseous phase from latex balloons. Environ Res 195:110700

Martellini T, Diletti G, Scortichini G, Lolini M, Lanciotti E, Katsoyiannis A, Cincinelli A (2016) Occurrence of polybrominated diphenyl ethers (PBDEs) in foodstuffs in Italy and implications for humans exposure. Food Chem Toxicol 89:32-38

Martinez-Bueno MJ, Gomez Ramos MJ, Bauer A, Fernandez-Alba AR (2019) An overview of non-targeted screening strategies based on high resolution accurate mass spectrometry for the identification of migrants coming from plastic food packaging materials. Trends Anal Chem 110:191-203

Mitchell G, Higgitt C, Gibson LT (2014) Emissions from polymeric materials: characterised by thermal desorption-gas chromatography. Polym Degrad Stabil 107:328-340

Motyakin MV, Schlick S (2006) ESR imaging and FTIR study of thermally treated poly(acrylonitrile-butadiene-styrene) (ABS) containing a hindered amine stabilizer: effect of polymermorphology, and butadiene and stabilizer content. Polym Degrad Stab 91:1462-1470

Munteanu SB, Vasile C (2005) Spectral and thermal characterization of styrene-butadiene copolymers with different architectures. J Optoelectron Adv Mater 7:3135-3148

Nekhoroshev VP, Popov EA, Nekhorosheva AV, Ruban SV (2005) Structural features of atactic polypropylene. Plast Massy 12:6-9

Noguchi M, Yamasaki A (2020) Volatile and semivolatile organic compound emissions from polymers used in commercial products during thermal degradation. Heliyon 6:e03314

Olmos D, Martin EV, Gonzalez-Benito J (2014) New molecular-scale information on polystyrene dynamics in PS and PS-BaTiO3 composites from FTIR spectroscopy. Phys Chem Chem Phys 16:24339-24349

Pei J, Yin Y, Cao J, Sun Y, Liu J, Zhang Y (2017) Time dependence of characteristic parameter for semi-volatile organic compounds (SVOCs) emitted from indoor materials. Build Environ 125:339-347

Pietron WJ, Warenik-Bany M, Wozniak B (2021) Polybrominated diphenyl ethers (PBDEs) in raw milk from different animal species and in infant formula Occurrence and risk assessment. Chemosphere 278:130479

Porubská M, Szöllős O, Kóňová A, Janigová I, Jašková M, Jomová K, Chodák I (2012) FTIR spectroscopy study of polyamide-6 irradiated by electron and proton beams. Polym Degrad Stab 97:523-531

Przybysz M, Marć M, Klein M, Reza Saeb M, Formela K (2018) Structural, mechanical and thermal behavior assessments of PCL/PHB blends reactively compatibilized with organic peroxides. Polym Test 67:513-521

Rahman F, Langford KH, Scrimshaw MD, Lester JN (2001) Polybrominated diphenyl ether (PBDE) flame retardants. Sci Total Environ 275(1-3):1-17
Royaux A, Fabre-Francke I, Balcar N, Barabant G, Bollard C, Lavedrine B, Cantin S (2017) Aging of plasticized polyvinyl chloride in heritage collections: the impact of conditioning and cleaning treatments. Polym Degrad Stabil 137:109-121

Santhoskumar AW, Palanivelu K, Sharma SK, Nayak SK (2010) A new synthesis of nickel 12-hydroxy oleate formulation to improve polyolefin's degradation. J Bioremed Biodegr 1:108

Sharkey M, Harrad S, Abdallah MAE, Drage DS, Berresheim H (2020) Phasing-out of legacy brominated flame retardants: the UNEP Stockholm convention and other legislative action worldwide. Environ Int 144:106041

Shittu O, Williams ID, Shaw PJ (2021) Global E-waste management: can WEEE make a difference? A review of e-waste trends, legislation, contemporary issues and future challenges. Waste Manage 120:549-563

Śmiełowska M, Zabiegała B (2020) Polybrominated diphenyl ether (PBDE) concentrations in dust from various indoor environments in Gdańsk, Poland: prediction of concentrations in indoor air and assessment of exposure of adults. Sci Total Environ 734:139437

Szczepańska N, Kudłak B, Namieśnik J (2018) Recent advances in assessing xenobiotics migrating from packaging material-a review. Anal Chim Acta 1023:1-21

Tang HP (2013) Recent development in analysis of persistent organic pollutants under the Stockholm convention. Trends Anal Chem 45:48-66

UNEP (2017) Draft guidance on sampling, screening and analysis of persistent organic pollutants in products and articles. Stockholm Convention, 58-59

Vuong AM, Yolton K, Poston KL, Xie C, Webster GM, Soejdin A, Braun JM, Dietrich KN, Lanphear BP, Chen A (2017) Prenatal and postnatal polybrominated diphenyl ether (PBDE) exposure and measures of inattention and impulsivity in children. Neurotoxicol Teratol 64:20-28

Vuong AM, Braun JM, Webster GM, Zoeller RT, Hoofnagle AN, Sjoedin A, Yolton K, Lanphear BP, Chen A (2018a) Polybrominated diphenyl ether (PBDE) exposures and thyroid hormones in children at age 3 years. Environ Int 117:339-347

Vuong AM, Yolton K, Dietrich KN, Braun JM, Lanphear BP, Chen A (2018b) Exposure to polybrominated diphenyl ethers (PBDEs) and child behavior: current findings and future directions. Horm Behav 101:94-104

Vuong AM, Yolton K, Poston KL, Xie C, Webster GM, Sjoedin A, Braun JM, Dietrich KN, Lanphear BP, Chen A (2018c) Childhood polybrominated diphenyl ether (PBDE) exposure and executive function in children in the HOME Study. Int J Hyg Environ Health 221:87-94

Wilke O, Jann O, Brçdner D (2004) VOC- and SVOC-emissions from adhesives, floor coverings and complete floor structures. Indoor Air 14:98-107

Wu Y, Xu Y, Wang D, Zhao Y, Weng S, Xu D, Wu J (2004) FT-IR spectroscopic investigation on the interaction between nylon 66 and lithium salts. J Appl Polym Sci 91:2869-2875

Wu JP, Wu SK, Tao L, She YZ, Chen XY, Feng WL, Zeng YH, Luo XJ, Mai BX (2020) Bioaccumulation characteristics of PBDEs and alternative brominated flame retardants in a wild frog-eating snake. Environ Pollut 258:113661

Xu W, Wang X, Cai Z (2013) Analytical chemistry of the persistent organic pollutants identified in the Stockholm convention: a review. Anal Chim Acta 790:1-13

Yanagisawa H, Kudo Y, Nakagawa K, Miyagawa H, Maruyama F, Fujimaki S (2018) Simultaneous screening of major flame retardants and plasticizers in polymer materials using pyrolyzer/thermal desorption gas chromatography mass spectrometry (Py/TD-GC-MS). Molecules 23(4):728-740

Yu J, Sun L, Ma C, Qiao Y, Yao H (2016) Thermal degradation of PVC: a review. Waste Manage 48:300-314 
Zacs D, Perkons I, Abudulajeva E, Pasecnaja E, Bartkiene E, Bartkevics V (2021) Polybrominated diphenyl ethers (PBDEs), hexabromocyclododecanes (HBCDD), dechlorane-related compounds (DRCs), and emerging brominated flame retardants (EBFRs) in foods: the levels, profiles, and dietary intake in Latvia. Sci Total Environ 752:141996

Zhang T, Zhou X, Xu A, Tian Y, Wang Y, Zhang Y, Gu Q, Wang S, Wang Z (2020) Toxicity of polybrominated diphenyl ethers (PBDEs) on rodent male reproductive system: a systematic review and meta-analysis of randomized control studies. Sci Total Environ $720: 137419$

Publisher's Note Springer Nature remains neutral with regard to jurisdictional claims in published maps and institutional affiliations. 